\title{
Pena-Shokeir Syndrome
}

National Cancer Institute

\section{Source}

National Cancer Institute. Pena-Shokeir Syndrome. NCI Thesaurus. Code C99008.

An autosomal recessive inherited disorder. It is characterized by arthrogryposis, facial anomalies, polyhydramnios, camptodactyly, intrauterine growth retardation, and pulmonary hypoplasia. Pulmonary hypoplasia is present in the vast majority of cases and is often the fatal component of this syndrome. 